\title{
Plant miRNA regulation is environmentally and developmentally-sensitive.
}

Patrick von Born ${ }^{1 \dagger}$ and Ignacio Rubio-Somoza ${ }^{1,2,}$

${ }^{1}$ Department of Molecular Biology, Max Planck Institute for Developmental Biology, 72076 Tübingen, Germany.

${ }^{2}$ Molecular Reprogramming and Evolution Laboratory, Centre for Research in Agricultural Genomics, 08193 Barcelona, Spain.

${ }^{\dagger}$ Current address: Department of Plant-Microbe Interactions, Max Planck Institute for Plant Breeding Research, Cologne, Germany.

* To whom correspondence should be addressed: ignacio.rubio@cragenomica.es

Running title:

Keywords: miRNA, Arabidopsis, translational inhibition, mRNA cleavage, temperature, age, development. 


\begin{abstract}
Development and fitness of any organism rely on properly controlled gene expression. This is especially true for plants, as their development is determined by both internal and external cues. MicroRNAs (miRNAs) are embedded in the genetic cascades that integrate and translate those cues into developmental programs. miRNAs negatively regulate their target genes mainly post-transcriptionally through two co-existing mechanisms; mRNA cleavage and translational inhibition. It is unclear whether the efficiency of miRNA-guided regulation is generally influenced by factors like ambient temperature or developmental stage. Here we show that plant miRNA accumulation, as well as miRNAs' mode of action can be temperature- and development-sensitive. Higher temperatures tend to induce a more pronounced accumulation of mature miRNAs. Both parameters have also an impact on the expression patterns of the core players involved in miRNA performance. We show that efficiency of miRNA-mediated gene silencing declines with age during vegetative development in a temperature-dependent manner. Co-existence of cleavage and translational inhibition was also found to be dependent on temperature and developmental stage. Therefore, each miRNA family specifically regulates their respective targets, while temperature and growth influence the performance of miRNA-dependent regulation in a more general way.
\end{abstract}




\section{Introduction}

Control of gene expression is paramount for any organism in order to exist and transit through different developmental stages as well as interrelate with their surroundings during their life cycle. All layers of control of gene expression are tightly regulated, from chromatin state to protein post-translational modifications, including mRNA stability. Small RNAs (sRNAs) have emerged in the last decades as central elements embedded in those regulatory layers. sRNAs come in several flavors depending on the source of RNA used for their biogenesis (1). MicroRNAs (miRNAs) are a special class of sRNAs that mainly regulate the expression of their targets post-transcriptionally. miRNA-dependent regulation has evolved independently in at least six eukaryotic lineages, including land plants (2). Most of the current knowledge about plant miRNA biogenesis, action and function comes from studies in the model organism Arabidopsis thaliana. Primary miRNA transcripts (primiRNA) arise from the RNA polymerase II-dependent expression of independent transcriptional units. Their expression pattern is under the control of specific regulatory sequences as is the case for protein coding genes (3). Pri-miRNAs are processed by the microprocessor complex in mature miRNA duplexes ranging from 19 to $24 \mathrm{nt}$ at the dicing bodies within the nuclei in a two-step enzymatic reaction (4). Proteins from the DICER family, mainly DICER-LIKE1 $(\mathrm{DCL} 1 ;(5))$ are the core components of the microprocessor complex and are assisted by accessory proteins such as HYPONASTIC LEAVES1 (HYL1; (6)) or DOUBLE-RNA BINDING PROTEIN 2 (DRB2;(7) SERRATE (SE; (8)) and CTERMINAL DOMAIN PHOSPHATASE-LIKE 1 (CPL1; (9)). The resulting mature miRNA duplexes are subsequently protected from degradation through HUA-ENHANCER 1 (HEN1)-mediated methylation (5). Next, HASTY (HST; (10)) participates in the transport of the stabilized miRNA duplexes to the cytoplasm where they are loaded into the RNAInduced Silencing Complex (RISC). Proteins from the ARGONAUTE (AGO) family are the main executive components of the RISC complex. The Arabidopsis genome has 10 AGO genes of which AGO1 (11) and AGO10 are considered the main players in posttranslational miRNA-mediated gene silencing (12). Once loaded into the RISC, one of the two duplex strands is degraded while the remaining one serves to scan the cytoplasm seeking for highly complementary mRNAs. miRNAs control the expression of their targets both by mRNA-target cleavage and translational inhibition (12). Beyond their existence, knowledge about the coexistence of both mechanisms in plants is scarce and suggests that the degree of their regulatory contribution might be cell-specific (13).

Plant miRNAs are involved in the regulation of a series of developmental and stressrelated genetic programs $(14,15)$. Nevertheless, little is known about whether general miRNA biogenesis and action, or the efficiency of their regulation change during the course of development and/or as consequence of environmental changes. Initial attempts of dealing with such a gap relied on assaying changes of endogenous miRNAs $(16,17)$. A major drawback from those studies is that mature miRNAs are usually produced from polygenic families and their accumulation is driven by distinct chromatin modifications, promoter activity and pri-miR structure (18-20).

In order to circumvent such limitations and clearly discern how those parameters might influence miRNA performance, we used an artificial and sensitive miRNA reporter (9). Our results show that accumulation of mature plant miRNAs and the resulting regulation (mechanism and efficiency) of their targets are dependent on growth temperature and developmental stage. We also show that both factors affect the expression of several key players involved both in miRNA biogenesis and action. 
The mechanisms of miRNA-mediated attenuation of protein expression have been harnessed to silence specific genes with artificial miRNAs (amiRs; $(21,22)$ ). Therefore, our findings are not just of relevance to understand miRNA regulation, but also instructive for the use of amiR-based gene silencing technology. 


\section{Material and Methods}

\section{Plant Material}

Plants were grown on soil in long days (16h light/8h dark) under a mixture of cool and warm white fluorescent light at $16^{\circ} \mathrm{C}$ and $23^{\circ} \mathrm{C}$ and $65 \%$ humidity. LUC miRNA-activity reporter (9) and rLUC control in which synonymous point mutations were introduced to render the firefly luciferase miRNA-insensitive (23) have been previously described.

RNA analyses

Total RNA was isolated as described in (24) using tissue pooled from 15 randomized individuals per sample and biological replicate.

Reverse transcription was performed with the RevertAid First Strand cDNA Synthesis Kit (Thermo Scientific) using 200ng of total RNA previously treated with DNase I (Thermo Scientific) following the protocol described in (25).

PCRs were carried out in presence of SYBR Green (Invitrogen) and monitored with the CFX384 Real-Time PCR Detection-System (Bio-Rad) in two technical and two biological replicates. Biological replicates were treated as independent samples. Relative expression changes were calculated using $2^{-\Delta \mathrm{Ct}}$ in all assays except in Fig. $3 A$ where the $2^{-\Delta \Delta \mathrm{Ct}}$ method was applied to normalize $L U C$ mRNA levels to the ones of $r L U C$. Expression levels were normalized to $\beta$-TUBULIN2 (At5g62690). Mature miRNA quantifications were performed by stem-loop RT-PCR as described (25).

For small RNA blots, $3 \mu \mathrm{g}$ of total RNA were used and two biological replicates performed. All primers used are listed in Table S1.

\section{Protein assays}

Proteins were isolated from the corresponding tissues from 15 randomized individuals per sample and biological replicate. After tissue homogenization, the resulting powder was resuspended in protein extraction buffer (PBS, Triton X-100 0.1\%, Complete EDTA-free (Roche)). After centrifugation, $50 \mu \mathrm{l}$ of protein were mixed with the same volume of BeetleJuice (PJK) Firefly substrate. Luciferase activity from two biological replicates was measured in technical triplicates on a Centro LB 960 (Berthold Technologies) device. Protein concentration of two biological replicates was assessed using the Bradford protein assay kit (BioRad) in technical triplicates. From this, Luciferase activity per $\mu \mathrm{g}$ of protein was calculated and the average of both biological replicates was used for further analysis. Values were normalized to the ones from rLUC. 


\section{Results}

\section{Addressing developmental and environmental influence on miRNA-mediated regulation}

RNA silencing has been described as an antiviral defense mechanism in both plants and invertebrates (26). Such defense mechanism is temperature sensitive with higher temperatures leading to increased production of virus-derived sRNAs (27). In order to study whether miRNA-mediated silencing is also under the influence of environment and/or development, we used an artificial miRNA reporter system that proved to be highly sensitive to perturbations in miRNA biogenesis and action (9). This reporter system relies on the expression of the Firefly luciferase gene $(L U C)$ under the constitutive Cauliflower Mosaic Virus 35S promoter. Simultaneously, the expression of an artificial miRNA (amiR$L U C)$ driven by the very same promoter, specifically silences $L U C$ expression. As control, we used a similar reporter system in which synonymous point mutations were introduced within the miRNA-complementary sequence in the LUC gene. Those point mutations rendered the LUC mRNA resistant ( $r L U C$ ) to amiR-LUC regulation (23). Using that artificial approach has clear advantages compared to rely on endogenous miRNAs. Among those advantages, the production of both miRNA and target are controlled by the same promoter and can be related at all growth conditions and developmental stages to the proper control allowing a fine dissection of all steps of the regulation.

Arabidopsis plants carrying either the $L U C$ or rLUC reporter systems were grown along at $16^{\circ} \mathrm{C}$ and $23^{\circ} \mathrm{C}$. $16^{\circ} \mathrm{C}$ is closer to the temperatures Arabidopsis typically experiences in its normal habitats, while $23^{\circ} \mathrm{C}$, despite being commonly used for Arabidopsis growth in controlled chambers, can be considered a stress temperature. Since the speed of Arabidopsis growth is temperature-dependent (28), we established discrete and equivalent time points to collect representative samples spanning the main developmental stages at both temperatures (Fig. 1A). Seedlings with the two first true leaves and leaves number 4 and 7 are representative of the transitions from juvenile to adult stages during vegetative development (Fig. 1B, (29)). We also assessed inflorescences containing all closed buds (stages 1 to $12(30)$ ) and pools of the three uppermost siliques after abscission of the senescent floral organs. Levels of the developmental timer miR156 were used to validate the equivalence of the samples collected at the two different growth conditions (31-33). As expected, miR156 accumulation declined as development progressed confirming that both sets of samples were developmentally equivalent (Fig. 1C). Slightly higher levels of miR156 observed in plants grown at lower temperatures are in line with former studies $(16,18)$.

\section{Mature miRNA accumulation has developmental and temperature-dependent components}

To study accumulation of mature amiR-LUC, we assayed amiR levels by stem-loop qRT-PCR (Fig. 2A) and small RNA blots (Fig. 2B).

Independent of growth temperature, amiR-LUC accumulated to higher levels in seedlings than at later stages during vegetative development, i.e. leaves 4 and 7 (Fig. 2A, Fig. 2B). Moreover, amiR-LUC levels were higher in siliques and inflorescences at $23^{\circ} \mathrm{C}$ when compared to vegetative organs (Fig. 2A, Fig. 2B).

Higher temperature was found to increase amiR-LUC levels in late vegetative development (leaf 7) and especially in inflorescence (Fig. 2A, Fig. 2B). 
Discrepancies found between the amiR-LUC levels determined either by qRT-PCR or small RNA blot in inflorescences and siliques might be explained by the intrinsic properties of both techniques (Fig. 2A, Fig. 2B). While stem-loop qRT-PCR monitors only the $21 \mathrm{nt}$ long species matching the designed amiR-LUC, small RNA blots can detect isoforms of different length and/or isoforms shifted by a few nucleotides (9).

A simple reason for miRNA accumulation being temperature and stage-dependent could be differential expression of factors involved in miRNA production. We therefore assayed whether the expression of core factors involved in miRNA biogenesis was regulated by development and/or growth temperature. We focused on the core executor DCL1 and in its assistants HYL1, DRB2, SERRATE and CPL1 (Fig. 3).

DCL1 mRNA expression levels were similar across all samples, with the exception of a marked increase in inflorescences from plants grown at $23^{\circ} \mathrm{C}$ compared to their counterparts grown at lower temperature (Fig. 3A). HYL1 and DRB2 showed similar expression levels in all tested tissues with a common trend of lower expression in vegetative tissues at $23^{\circ} \mathrm{C}$ (Fig. 3B, Fig. 3C). Similar to DCL1, DRB2 levels were increased in inflorescences from plants grown at $23^{\circ} \mathrm{C}$ (Fig. $3 \mathrm{C}$ ).

$S E$ was more highly expressed in vegetative than in reproductive organs in plants grown at $16^{\circ} \mathrm{C}$ (Fig. 3D). As for DCL1 and DRB2, SE expression levels in reproductive tissues were higher at $23^{\circ} \mathrm{C}$.

Plants grown at $23^{\circ} \mathrm{C}$ presented the same trend of lower levels of CPL1 expression in vegetative tissues that was also found for $H Y L 1$ and DRB2 (Fig. 3E). While its expression at $16^{\circ} \mathrm{C}$ did not change during vegetative development, it gradually increased in plants grown at $23^{\circ} \mathrm{C}$ reaching highest values in leaf 7 (Fig. 3E).

Collectively, our results show dynamic and heterogeneous expression profiles of different members of the core miRNA biogenesis machinery. We observed little correlation between these patterns and the accumulation of mature amiR-LUC across the different samples with the only exception of inflorescences from plants grown at $23^{\circ} \mathrm{C}$. When compared to plants grown at $16^{\circ} \mathrm{C}$, higher levels of amiR-LUC were paralleled by higher levels of DCL1, DRB2, SE and CPL1. Further, the slightly lower levels of expression for $H Y L 1$ coincide with DRB2 expression levels during vegetative development at $23^{\circ} \mathrm{C}$.

It is noteworthy that for most of the miRNA biogenesis players, we observed a general tendency to higher expression levels in vegetative organs at $16^{\circ} \mathrm{C}$ than in plants grown at $23^{\circ} \mathrm{C}$, while the opposite was true in reproductive organs.

\section{Efficiency and mode of action of miRNA-mediated regulation is temperature dependent}

Once we had established that development and temperature affect the accumulation of mature miRNAs, we sought to explore whether miRNA-mediated gene silencing was also developmentally and environmentally regulated.

We firstly assayed the contribution of target cleavage regulation in response to different growth temperatures and across development. LUC mRNA levels were assayed in the same samples used for qRT-PCR and with primers flanking the miRNA-targeted sequence. $L U C$ levels were reduced by 60 to $85 \%$ when compared to rLUC depending on tissue and growth conditions (Fig. 4A). We found that generally, higher levels of mature amiR-LUC (Fig. 2) lead to lower levels of LUC transcripts (Fig. 4A). Thus, seedlings and inflorescences from plants grown at $16^{\circ} \mathrm{C}$ presented higher levels of $L U C$ transcripts than 
their counterparts grown at $23^{\circ}$ (Fig. 4A). During vegetative development, we observed an increase in LUC mRNA levels between seedlings and leaf 4 both at $16^{\circ} \mathrm{C}$ and $23^{\circ} \mathrm{C}$ (Fig. 4A).

To assess the contribution of translational inhibition we inferred the levels of LUC protein by measuring LUC activity in protein extracts from samples collected at the same time as the ones used for expression assays (Fig. 4B). We observed that during vegetative development LUC levels gradually increased, although to a different extent, both at $16^{\circ} \mathrm{C}$ and $23^{\circ} \mathrm{C}$ (Fig. 4B). We also found that the ultimate effect of miRNA-dependent regulation over the production of functional targeted-protein was temperature dependent. LUC protein levels were clearly higher at $16^{\circ} \mathrm{C}$ than the ones found in samples from plants grown at $23^{\circ} \mathrm{C}$ in leaf 7 , inflorescences and siliques (Fig. 4B).

Next, we studied whether the differential contribution of both regulatory mechanisms was developmentally and/or environmentally determined. We reasoned that translational inhibition mechanisms would lead to a further reduction of LUC protein levels when compared to mRNA levels. Therefore, we created a Coexistence index, representing the ratio between LUC protein levels and LUC mRNA levels. Values higher than 1 indicated a low contribution from translational inhibition to miRNA-dependent regulation, while the opposite was true for values smaller than 1. As seen in Fig. 4C, the translational inhibition mechanism was gradually less effective during vegetative development at $16^{\circ} \mathrm{C}$. We observed the same tendency in leaf 7 from plants grown at $23^{\circ} \mathrm{C}$ when compared with earlier stages of development (seedlings and leaf 4). In inflorescences and siliques, translational inhibition was more potent at $23^{\circ} \mathrm{C}$ when compared to $16^{\circ} \mathrm{C}$.

The two main effectors within miRNA-loaded RISC complexes are AGO1 and AGO10. Both proteins have redundant but also specific roles in miRNA-mediated gene silencing (34). Thus, it has been suggested that AGO10 has a more prominent role on translational inhibition (34) despite evidence that it is also able to cleave its mRNA targets (35). To ascertain whether developmental and environmentally-dependent changes on the coexistence index correlated with variations on their expression, we analyzed both AGO1 and AGO10 profiles by qRT-PCR (Fig. 4D; Fig. 4E). Interestingly, during vegetative development both $A G O 1$ and $A G 010$ presented an opposite expression pattern. AGO1 expression was consistently higher at $16^{\circ} \mathrm{C}$ while the opposite was true for AGO10. Nevertheless, we did not observe any correlation between AGO1 and AGO10 expression patterns and the differences in co-existence index.

Altogether, these results show that developmental as well as environmental components influence both miRNA regulation and the balance between cleavage and translational inhibition mechanisms of gene silencing. 


\section{Discussion}

Our findings show that plant miRNA performance (accumulation, efficiency and coexistence of target cleavage and translational inhibition) is influenced by both development and environment. Our results support that the expression of several central players in miRNA performance also depends on development and temperature in which plants are grown.

The view of the different pathways involved in sRNA production and action was initially rather simplistic and static (36). It was generally assumed that molecular players devoted to generate each type of sRNA were ubiquitously expressed and, therefore, the main layer of control on sRNA-mediated regulation was orchestrated by the expression patterns of the RNA from which they originated. We are currently starting to appreciate that this might be a more dynamic process (37). Our results support a more dynamic scenario in which the expression of molecular players and mechanisms involved in miRNA-mediated gene silencing are developmentally and environmentally-sensitive.

Although siRNA biogenesis in plants has been reported to be temperature sensitive, with siRNA levels correlating with growth temperature, mature miRNA accumulation has been thought to be largely temperature insensitive $(27,38,39)$. In contrast to studies where whole plants were assayed, our study dissects the temperature effect using discrete samples that encompass the different developmental stages during vegetative and reproductive development. Our analysis shows that amiR-LUC accumulation is temperature-responsive in leaves produced at later stages (leaf 7) when compared to an early phase of vegetative development (leaf 4). That positive temperature effect on amiRLUC levels is more dramatic in reproductive development with a greater accumulation in inflorescences grown at $23^{\circ} \mathrm{C}$ (Fig. 2A; Fig. 2B). Such increased accumulation is likely to be a consequence of the higher expression of the central miRNA biogenesis factor DCL1 (Fig. 3A) and its assistants DRB2 (Fig. 3C) and SE (Fig. 3D). Among the factors involved in miRNA biogenesis, temperature had a clear effect on the expression of SE and CPL1 which are additionally involved in more general processes such as mRNA splicing, which seems to be also temperature dependent (40-43).

miRNA-mediated gene silencing relies on two mechanisms that are thought to coexist, target cleavage and translational inhibition (12). Nevertheless, beyond their existence little is known about their individual contribution to target gene silencing in plants. In mammals, miRNA-mediated regulation occurs mainly through target degradation regardless of cell type, growth conditions or translational state $(44,45)$. Initial work shows that in plants the contribution of both mechanisms is cell-specific in pollen (13). It is also unknown whether environmental conditions can influence plant miRNA efficiency and their mode of action.

Our results reveal that the efficiency of miRNA regulation decays with age in Arabidopsis (Fig. 4C; leaf 4 versus leaf 7) with this decrease being more evident at low temperatures. Likewise, the contribution of translational inhibition also decreases with age showing a similar discrepancy between mRNA and protein levels to the one found in mutant plants impaired in that mechanism of gene silencing (12). In contrast, the contribution from this mechanism increases with temperature during reproductive development (Fig. 4C). Although we found an opposite effect of temperature on the expression of the two main silencing effectors, AGO1 and AGO10, we did not find any correlation between their levels and the difference in miRNA efficiency or mode of action. It has been suggested that miR168-loaded AGO10 negatively regulates AGO1 protein levels without affecting its mRNA levels (34). Our results suggest that AGO10 might also affect AGO1 mRNA levels. 
According to our results, miRNA regulation is more efficient at higher temperatures. Therefore, it is tempting to hypothesize miR168 AG010-loaded contributing to AGO1 mRNA degradation at $23^{\circ} \mathrm{C}$. In support of such hypothesis, it has been described that AGO10 has slicing activity (35). This point deserves further investigation.

It has been recently suggested that the DCL1 partner proteins HYL1 and DRB2 determine whether a miRNA triggers cleavage or translational repression of its targeted mRNAs (46). Thus, while HYL1-mediated miRNA production contributes to degradation of the targeted mRNA, DRB2-dependent miRNA biogenesis triggers translational inhibition. Despite the observed changes in the coexistence between both regulatory mechanisms over development, we could only correlate higher levels of DRB2 expression to a more pronounced contribution through translational inhibition in inflorescences grown at $23^{\circ} \mathrm{C}$ when compared to lower temperature (Fig. 2C, Fig. 4C). Absence of correlation suggests that additional players and/or posttranslational modifications of the already known ones might determine the mechanism through which miRNAs regulate the expression of their targets $(9,47)$. Therefore, in contrast to animals, plant miRNA performance depends on the cell-type, developmental stage and growth conditions.

Plants compromised in essential components of the miRNA machinery, such as DCL1 and AGO1 $(48,49)$, are usually sterile when grown at $23^{\circ} \mathrm{C}$. Nevertheless, a partial restoration of fertility is found when those plants are grown at lower temperatures. According to our results, miRNA regulation efficiency in inflorescences is lower at $16^{\circ} \mathrm{C}$ when compared to plants grown at higher temperatures. Consequently, miRNA gene silencing might play a minor role in the general regulation of gene expression at low temperatures in inflorescence thereby explaining fertility restoration in these growth conditions.

Finally, our results are informative for the use of artificial miRNAs to downregulate endogenous genes at late stages of development or as part of crop protection strategies. 


\section{Funding}

Work at the Max Planck Institute in the Department of Molecular Biology was supported by the Max Planck Society and DFG SFB1101. I. R-S. is supported by the Spanish Ministry of Economy and Competitiveness (BFU2014-58361-JIN and through the "Severo Ochoa Programme for Centres of Excellence in R\&D" 2016-2019 (SEV-2015-0533)) and the CERCA programme from the Generalitat de Catalunya..

\section{Acknowledgments}

We greatly acknowledge the support from Detlef Weigel since majority substantial part of this work was conducted at the MPI for Developmental Biology. We thank Silvio Collani, Catia Igreja and Elisa Izaurralde for support with establishing the luciferase quantification assay, and Patricia Lang, Sascha Laubinger, Peter Brodersen, Pablo Manavella and members of the MoRE Lab for critical reading of the manuscript.

\section{Contributions}

I.R-S. designed the research, performed experiments, analyzed the data, and wrote the manuscript. P.v.B. performed experiments, analyzed data and helped in manuscript drafting. 


\section{Bibliography}

1. Axtell MJ (2013) Classification and comparison of small RNAs from plants. Annu Rev Plant Biol 64:137-159.

2. Tarver JE, et al. (2015) microRNAs and the evolution of complex multicellularity: identification of a large, diverse complement of microRNAs in the brown alga Ectocarpus. Nucleic Acids Res 43(13):6384-6398.

3. Voinnet O (2009) Origin, biogenesis, and activity of plant microRNAs. Cell 136(4):669-687.

4. Fang Y \& Spector DL (2007) Identification of nuclear dicing bodies containing proteins for microRNA biogenesis in living Arabidopsis plants. Curr Biol 17(9):818-823.

5. Park W, Li J, Song R, Messing J, \& Chen X (2002) CARPEL FACTORY, a Dicer homolog, and HEN1, a novel protein, act in microRNA metabolism in Arabidopsis thaliana. Curr Biol 12(17):1484-1495.

6. Han MH, Goud S, Song L, \& Fedoroff N (2004) The Arabidopsis double-stranded RNAbinding protein HYL1 plays a role in microRNA-mediated gene regulation. Proc Natl Acad Sci U S A 101(4):1093-1098.

7. Eamens AL, Kim KW, Curtin SJ, \& Waterhouse PM (2012) DRB2 is required for microRNA biogenesis in Arabidopsis thaliana. PLoS One 7(4):e35933.

8. Yang L, Liu Z, Lu F, Dong A, \& Huang H (2006) SERRATE is a novel nuclear regulator in primary microRNA processing in Arabidopsis. Plant J 47(6):841-850.

9. Manavella PA, et al. (2012) Fast-forward genetics identifies plant CPL phosphatases as regulators of miRNA processing factor HYL1. Cell 151(4):859-870.

10. Park MY, Wu G, Gonzalez-Sulser A, Vaucheret H, \& Poethig RS (2005) Nuclear processing and export of microRNAs in Arabidopsis. Proc Natl Acad Sci U S A 102(10):3691-3696.

11. Fagard M, Boutet S, Morel JB, Bellini C, \& Vaucheret H (2000) AGO1, QDE-2, and RDE-1 are related proteins required for post-transcriptional gene silencing in plants, quelling in fungi, and RNA interference in animals. Proc Natl Acad Sci U S A 97(21):11650-11654.

12. Brodersen $P$, et al. (2008) Widespread translational inhibition by plant miRNAs and siRNAs. Science 320(5880):1185-1190.

13. Grant-Downton R, et al. (2013) Artificial microRNAs reveal cell-specific differences in small RNA activity in pollen. Curr Biol 23(14):R599-601.

14. Rubio-Somoza I \& Weigel D (2011) MicroRNA networks and developmental plasticity in plants. Trends Plant Sci 16(5):258-264.

15. Sunkar R, Li YF, \& Jagadeeswaran G (2012) Functions of microRNAs in plant stress responses. Trends Plant Sci 17(4):196-203.

16. Lee $\mathrm{H}$, et al. (2010) Genetic framework for flowering-time regulation by ambient temperature-responsive miRNAs in Arabidopsis. Nucleic Acids Res 38(9):3081-3093.

17. May P, et al. (2013) The effects of carbon dioxide and temperature on microRNA expression in Arabidopsis development. Nat Commun 4:2145.

18. Kim W, et al. (2016) Structural determinants of miR156a precursor processing in temperature-responsive flowering in Arabidopsis. J Exp Bot 67(15):4659-4670.

19. Pico S, Ortiz-Marchena MI, Merini W, \& Calonje M (2015) Deciphering the Role of POLYCOMB REPRESSIVE COMPLEX1 Variants in Regulating the Acquisition of Flowering Competence in Arabidopsis. Plant Physiol 168(4):1286-1297.

20. $\mathrm{Xu} \mathrm{M}$, et al. (2016) Developmental Functions of miR156-Regulated SQUAMOSA PROMOTER BINDING PROTEIN-LIKE (SPL) Genes in Arabidopsis thaliana. PLoS Genet 12(8):e1006263. 
21. Parizotto EA, Dunoyer P, Rahm N, Himber C, \& Voinnet O (2004) In vivo investigation of the transcription, processing, endonucleolytic activity, and functional relevance of the spatial distribution of a plant miRNA. Genes Dev 18(18):2237-2242.

22. Schwab R, Ossowski S, Riester M, Warthmann N, \& Weigel D (2006) Highly specific gene silencing by artificial microRNAs in Arabidopsis. Plant Cell 18(5):1121-1133.

23. Karlsson P, et al. (2015) KH domain protein RCF3 is a tissue-biased regulator of the plant miRNA biogenesis cofactor HYL1. Proc Natl Acad Sci U S A 112(45):14096-14101.

24. Box MS, Coustham V, Dean C, \& Mylne JS (2011) Protocol: A simple phenol-based method for 96-well extraction of high quality RNA from Arabidopsis. Plant Methods 7:7.

25. Varkonyi-Gasic E, Wu R, Wood M, Walton EF, \& Hellens RP (2007) Protocol: a highly sensitive RT-PCR method for detection and quantification of microRNAs. Plant Methods 3:12.

26. Ding SW \& Voinnet O (2007) Antiviral immunity directed by small RNAs. Cell 130(3):413426.

27. Szittya G, et al. (2003) Low temperature inhibits RNA silencing-mediated defence by the control of siRNA generation. EMBO J 22(3):633-640.

28. Capovilla G, Schmid M, \& Pose D (2015) Control of flowering by ambient temperature. J Exp Bot 66(1):59-69.

29. Huijser P \& Schmid M (2011) The control of developmental phase transitions in plants. Development 138(19):4117-4129.

30. Smyth DR, Bowman JL, \& Meyerowitz EM (1990) Early flower development in Arabidopsis. Plant Cell 2(8):755-767.

31. Rubio-Somoza l, et al. (2014) Temporal control of leaf complexity by miRNA-regulated licensing of protein complexes. Curr Biol 24(22):2714-2719.

32. Wang JW, Czech B, \& Weigel D (2009) miR156-regulated SPL transcription factors define an endogenous flowering pathway in Arabidopsis thaliana. Cell 138(4):738-749.

33. Wu G, et al. (2009) The sequential action of miR156 and miR172 regulates developmental timing in Arabidopsis. Cell 138(4):750-759.

34. Mallory AC, et al. (2009) Redundant and specific roles of the ARGONAUTE proteins AGO1 and ZLL in development and small RNA-directed gene silencing. PLoS Genet 5(9):e1000646.

35. Ji L, et al. (2011) ARGONAUTE10 and ARGONAUTE1 regulate the termination of floral stem cells through two microRNAs in Arabidopsis. PLoS Genet 7(3):e1001358.

36. Reis RS, Eamens AL, \& Waterhouse PM (2015) Missing Pieces in the Puzzle of Plant MicroRNAs. Trends Plant Sci 20(11):721-728.

37. Achkar NP, Cambiagno DA, \& Manavella PA (2016) miRNA Biogenesis: A Dynamic Pathway. Trends Plant Sci 21(12):1034-1044.

38. Niu QW, et al. (2006) Expression of artificial microRNAs in transgenic Arabidopsis thaliana confers virus resistance. Nat Biotechnol 24(11):1420-1428.

39. Szittya G \& Burgyan J (2013) RNA interference-mediated intrinsic antiviral immunity in plants. Curr Top Microbiol Immunol 371:153-181.

40. Chen T, et al. (2013) A KH-domain RNA-binding protein interacts with FIERY2/CTD phosphatase-like 1 and splicing factors and is important for pre-mRNA splicing in Arabidopsis. PLoS Genet 9(10):e1003875.

41. Laubinger $\mathrm{S}$, et al. (2008) Dual roles of the nuclear cap-binding complex and SERRATE in pre-mRNA splicing and microRNA processing in Arabidopsis thaliana. Proc Natl Acad Sci U $S$ A 105(25):8795-8800.

42. Pose D, et al. (2013) Temperature-dependent regulation of flowering by antagonistic FLM variants. Nature 503(7476):414-417. 
43. Seo PJ, Kim MJ, Ryu JY, Jeong EY, \& Park CM (2011) Two splice variants of the IDD14 transcription factor competitively form nonfunctional heterodimers which may regulate starch metabolism. Nat Commun 2:303.

44. Eichhorn SW, et al. (2014) mRNA destabilization is the dominant effect of mammalian microRNAs by the time substantial repression ensues. Mol Cell 56(1):104-115.

45. Guo H, Ingolia NT, Weissman JS, \& Bartel DP (2010) Mammalian microRNAs predominantly act to decrease target mRNA levels. Nature 466(7308):835-840.

46. Reis RS, Hart-Smith G, Eamens AL, Wilkins MR, \& Waterhouse PM (2015) Gene regulation by translational inhibition is determined by Dicer partnering proteins. Nat Plants 1:14027.

47. Cho SK, Ryu MY, Shah P, Poulsen CP, \& Yang SW (2016) Post-Translational Regulation of miRNA Pathway Components, AGO1 and HYL1, in Plants. Mol Cells 39(8):581-586.

48. Bohmert K, et al. (1998) AGO1 defines a novel locus of Arabidopsis controlling leaf development. EMBO J 17(1):170-180.

49. Field S \& Thompson B (2016) Analysis of the Maize dicer-like1 Mutant, fuzzy tassel, Implicates MicroRNAs in Anther Maturation and Dehiscence. PLoS One 11(1):e0146534.

\section{Figure legends}

Fig. 1. Addressing developmental and environmental influence on miRNA-mediated regulation via a Luciferase reporter system. (A) Discrete time points for tissue collection over Arabidopsis life cycle at $16^{\circ} \mathrm{C}$ (blue) and $23^{\circ} \mathrm{C}$ (green). (B) Representative pictures of the different leaf stages collected spanning vegetative development. Arrows point to the collected leaves. (C) Mature miR156 qRT-PCR to ensure that samples from both datasets $\left(16^{\circ} \mathrm{C}\right.$ and $\left.23^{\circ} \mathrm{C}\right)$ were at equivalent developmental points. Black dots represent one biological replicate each, calculated from two technical replicates. Lines, (blue $=16^{\circ} C$, green $=23^{\circ} \mathrm{C}$ ) represent the average between two biological replicates. "Inflores" stands for inflorescences.

Fig. 2. AmiR-LUC accumulation is developmentally and temperature-dependent. (A) Mature amiR-LUC accumulation assayed by $q R T-P C R$. Black dots represent one biological replicate each calculated from two technical replicates. Lines, (blue $=16^{\circ} \mathrm{C}$, green $=23^{\circ} \mathrm{C}$ ) represent the average between two biological replicates. "Inflores" stands for inflorescences. (B) Representative sRNA blot for amiR-LUC accumulation.

Fig. 3 Effect of development and temperature on the expression of miRNA biogenesis factors. (A) DCL1. (B) HYL1. (C) DRB2. (D) SE. (E) CPL1. Black dots represent one biological replicate each calculated from two technical replicates. Lines, (blue $=16^{\circ} C$, green $=23^{\circ} \mathrm{C}$ ) represent the average between two biological replicates. "Inflores" stands for inflorescences.

Fig. 4. miRNA mode of action is developmentally and temperature-dependent. (A) LUC mRNA expression levels assayed by qRT-PCR normalized to LUC mRNA in rLUC control plants (red dotted line). Lines, (blue $=16^{\circ} \mathrm{C}$, green $=23^{\circ} \mathrm{C}$ ) represent the average between two biological replicates. (B) LUC protein levels. Black dots represent one biological replicate each calculated from two technical replicates. Lines, (blue $=16^{\circ} \mathrm{C}$, green $=23^{\circ} \mathrm{C}$ ) represent the average between two biological replicates normalized to LUC protein levels in rLUC control plants (red dotted line). (C) Coexistence index is the ratio of average protein levels by average mRNA levels from each sample and condition. (D) AGO1 expression levels assayed by $q R T-P C R$. Black dots represent one biological replicate 
each calculated from two technical replicates. Lines, (blue $=16^{\circ} \mathrm{C}$, green $=23^{\circ} \mathrm{C}$ ) represent the average between two biological replicates. (E) AGO10 expression levels assayed by qRT-PCR. Black dots represent one biological replicate each calculated from two technical replicates. Lines, (blue $=16^{\circ} \mathrm{C}$, green $=23^{\circ} \mathrm{C}$ ) represent the average between two biological replicates. (A-E) "Inflores" stands for inflorescences. 
A

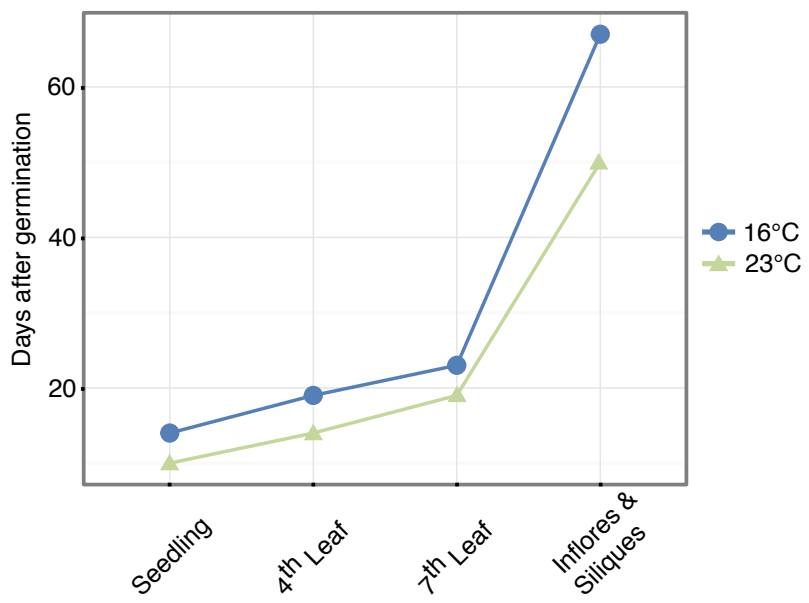

B

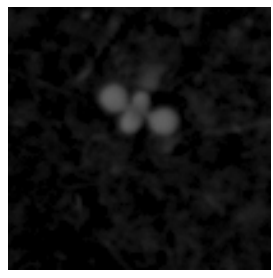

Seedling

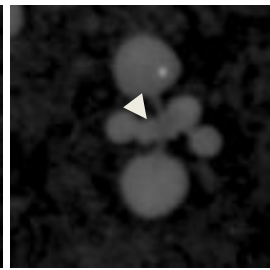

$4^{\text {th }}$ Leaf

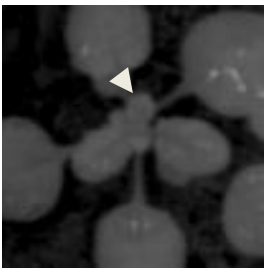

$7^{\text {th }}$ Leaf
C

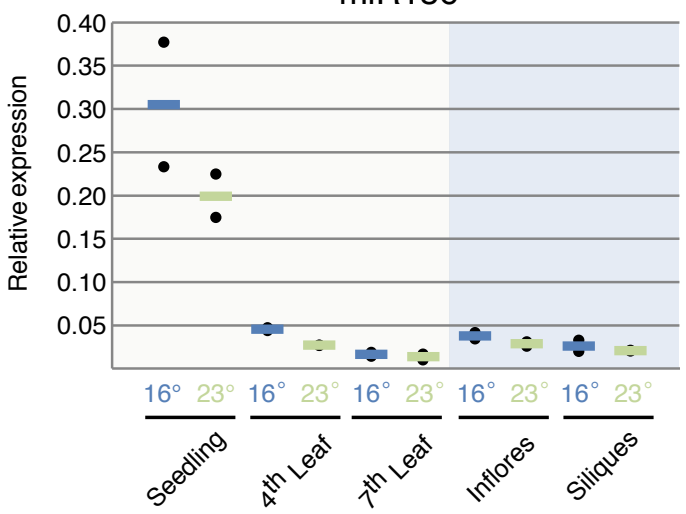



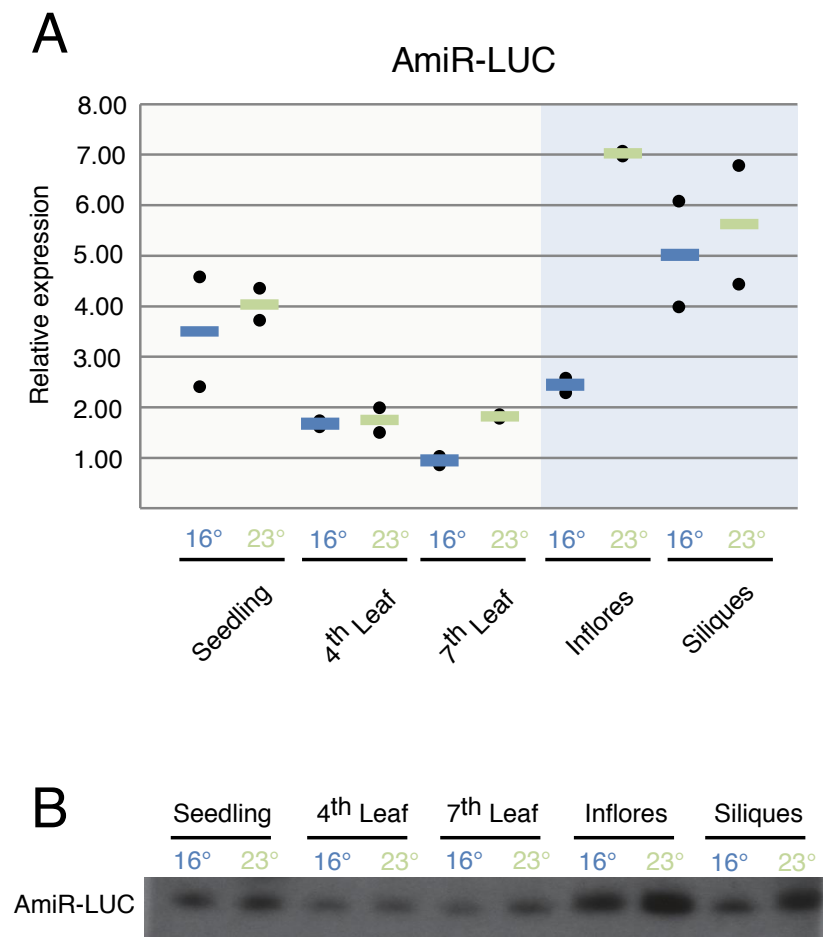

U6 
A

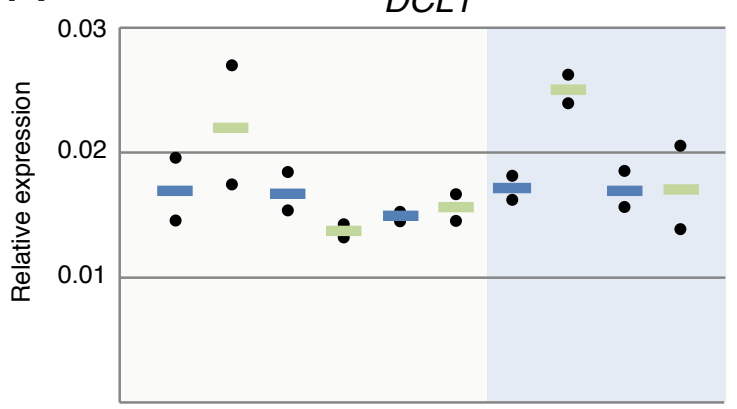

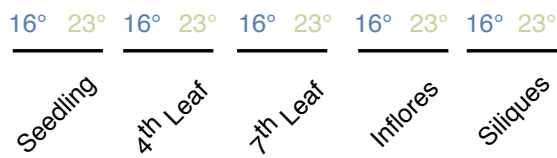

C

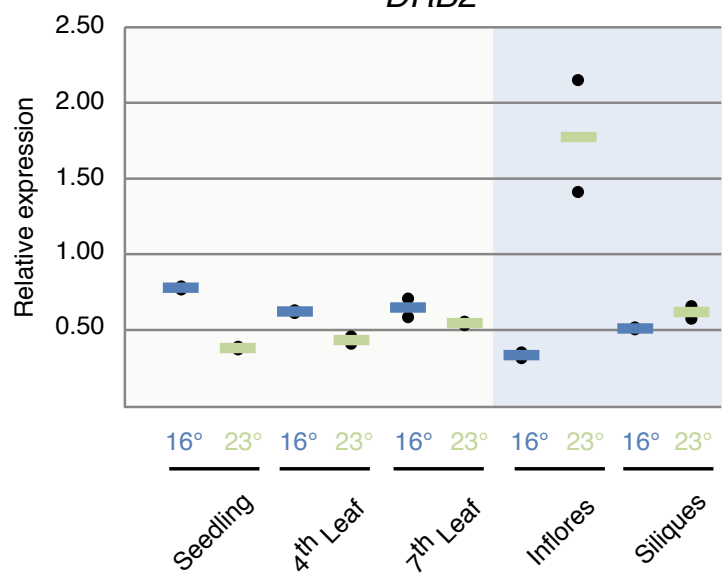

B

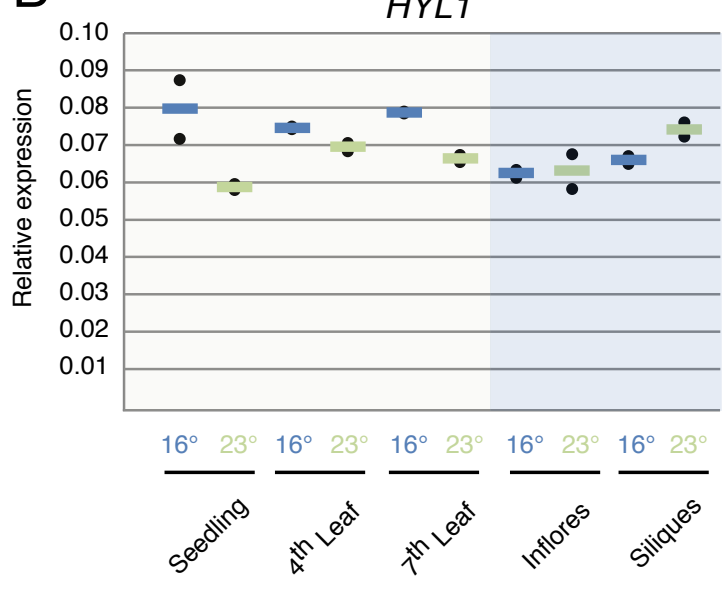

D

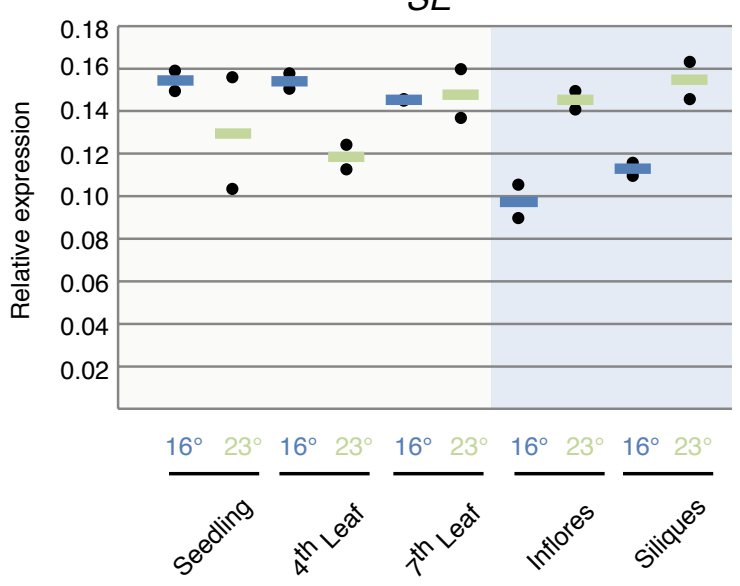

E

CPL1

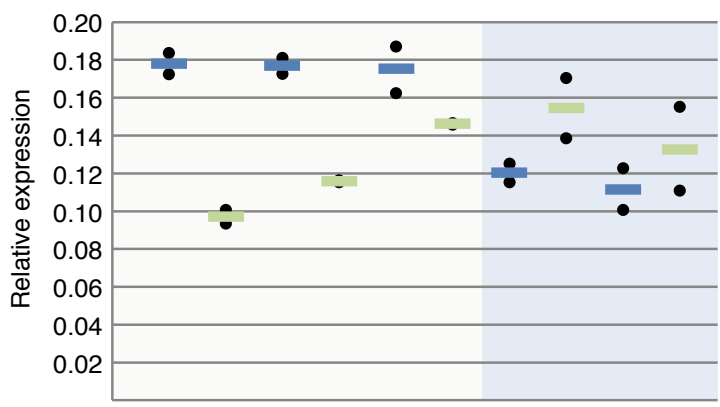

$\underline{16^{\circ} 23^{\circ}} \underline{16^{\circ} 23^{\circ}} \underline{16^{\circ} 23^{\circ}} \underline{16^{\circ} 23^{\circ}} \underline{16^{\circ} 23^{\circ}}$

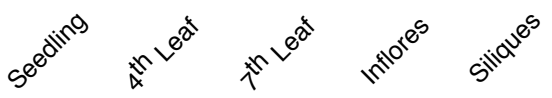


A

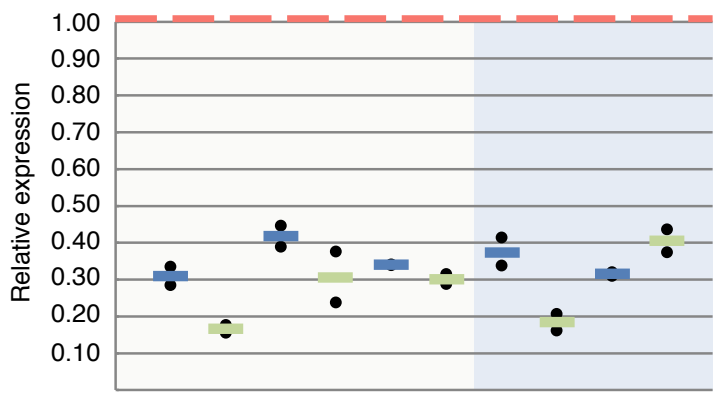

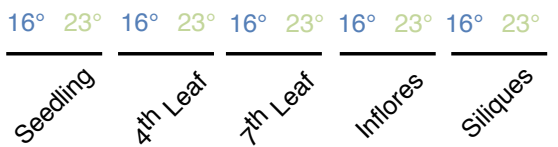

C LUC Protein / LUC mRNA

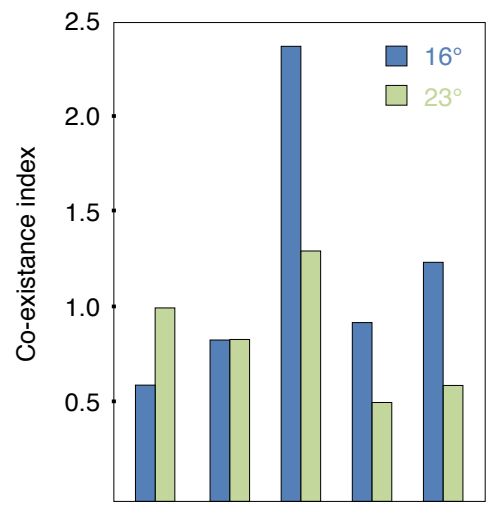

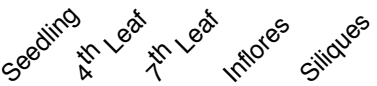

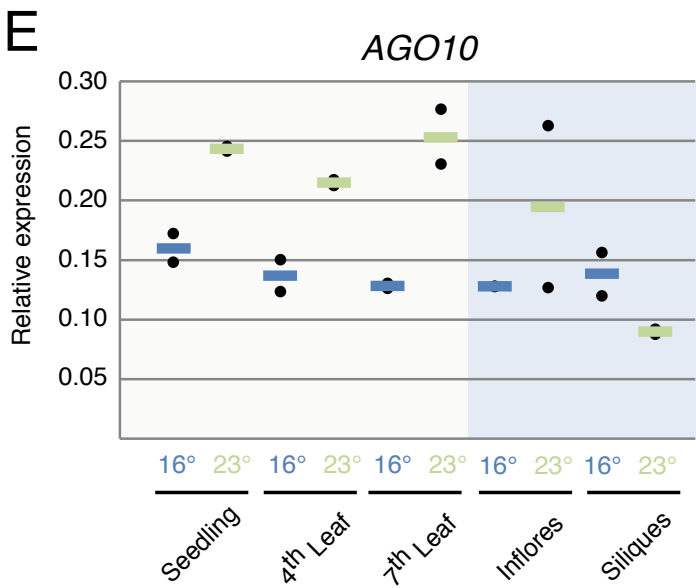

B

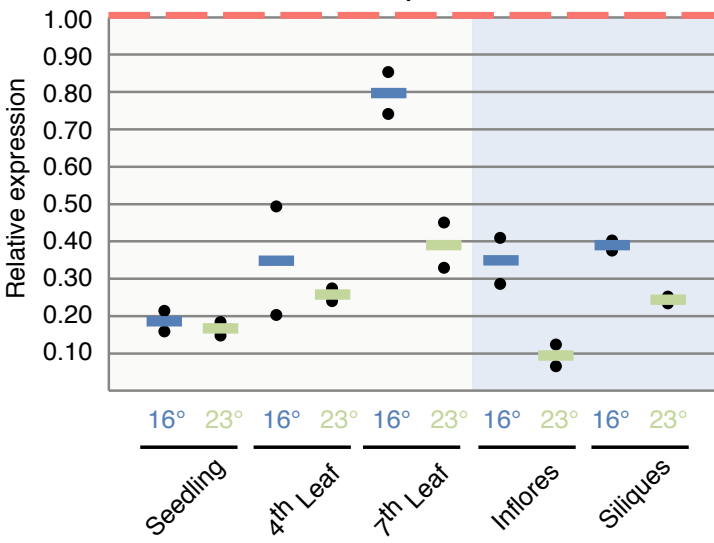

D

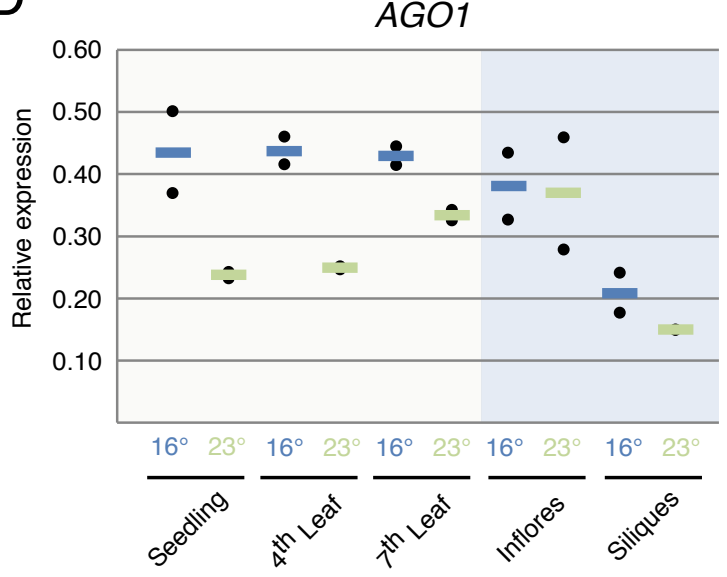

